\title{
Diabetes medications with cardiovascular protection: the likelihood of benefit from combination therapy increases further following new evidence during 2020
}

\author{
ROBERT EJ RYDER, ${ }^{1}$ MUHAMMAD A ABDUL-GHANI, ${ }^{2}$ RALPH A DEFRONZO ${ }^{2}$
}

Key words: Type 2 diabetes, cardiovascular outcome studies, pioglitazone, SGLT2 inhibitors, GLP-1 receptor agonist, heart failure, stroke

In every recent year, new cardiovascular outcome studies are published, illuminating our understanding regarding diabetes medications with cardiovascular protection, and we have discussed these in our previous editorials. ${ }^{1-6}$ In 2020 two new studies from the sodium glucose transporter 2 (SGLT2) inhibitor class and one from the glucagon-like peptide-1 receptor agonist (GLP-1RA) class of antidiabetic medications are worth highlighting. Each provided new information to help our understanding about the cardioprotective benefits of these classes so that we can further improve patient care. On 16 June 2020, during the 80th Scientific Sessions of the American Diabetes Association virtual meeting, the results of the Evaluation of Ertugliflozin Efficacy and Safety Cardiovascular Outcomes Trial (VERTIS CV) study were presented and have since been published in the New England Journal of Medicine.7,8 On 29 August 2020, during the European Society of Cardiology - The Digital Experience Congress 2020, the results of the Empagliflozin Outcome Trial in Patients with Chronic Heart Failure and a Reduced Ejection Fraction (EMPEROR-Reduced) were presented and published simultaneously in the New England Journal of Medicine.9,10 The study was then presented in detail on 24 September 2020 during the European Association for the Study of Diabetes Virtual Congress (EASD) 2020. ${ }^{11}$ With regard to the GLP-1RA class, some further cardiovascular outcome data were presented at the EASD 2020 in the form of a post hoc analysis of pooled data from the LEADER, SUSTAIN 6 and PIONEER 6 cardiovascular outcome studies. ${ }^{12}$

\section{SGLT2 inhibitors}

VERTIS CV was a randomised controlled trial of the SGLT2 inhibitor ertugliflozin versus placebo in 8,246 people with type 2 diabetes all of whom had prior cardiovascular disease. ${ }^{7,8}$ After a follow-up

City Hospital, Birmingham, UK

University of Texas Health Science Center, San Antonio, Texas, USA

Address for correspondence: Dr Bob Ryder

Diabetes and Endocrine Unit, City Hospital, Dudley Road, Birmingham B18 7QH, UK

E-mail: bob.ryder@nhs.net

Br J Diabetes 2020;20:84-88

https://doi.org/10.15277/bjd.2020.276 period of 6.1 years, the primary endpoint of 3-point major adverse cardiovascular events (MACE: cardiovascular death, non-fatal myocardial infarction and non-fatal stroke) achieved statistical significance for non-inferiority $(\mathrm{HR}=0.97,95.6 \% \mathrm{Cl} 0.85$ to 1.11 ; $\mathrm{p}<0.001$ for non-inferiority). ${ }^{7,8}$ However, it did not achieve statistical significance for superiority with regard to 3-point MACE or the combined endpoint of death from cardiovascular causes or hospitalisation for heart failure (HHF). There was a 30\% reduction in HHF ( $\mathrm{HR}=0.70,95 \% \mathrm{Cl} 0.54$ to 0.90$){ }^{7,8}$

EMPEROR-Reduced was a randomised controlled trial of empagliflozin $10 \mathrm{mg}$ daily versus placebo in 3,730 patients with class II, III or IV heart failure and an ejection fraction of $40 \%$ or less (HFrEF). ${ }^{9-11} 50 \%$ of the study population had type 2 diabetes and $50 \%$ did not have diabetes. Over a median follow-up of 16 months there was a $25 \%$ reduction in the primary composite endpoint of cardiovascular death or hospitalisation for worsening heart failure $(\mathrm{HR}=0.75,95 \% \mathrm{Cl} 0.65$ to 0.86$)$. The results were similar whether the patient had diabetes ( $\mathrm{HR}=0.72,95 \% \mathrm{Cl} 0.60$ to 0.87 ) or did not have diabetes ( $\mathrm{HR}=0.78,95 \% \mathrm{Cl} 0.64$ to 0.97$)$. There was a $30 \%$ reduction in $\mathrm{HHF}(\mathrm{HR}=0.70,95 \% \mathrm{Cl} 0.58$ to 0.85$){ }^{9-11}$

Building on the experience gained from their predecessor trials with SGLT2 inhibitors, $1,3,5,6,13,14$ we have learned further from VERTIS-CV and EMPORER-Reduced about the extent to which the cardiovascular benefits of SGLT2 inhibitors are mediated through the protection from heart failure. Figure $1 \mathrm{~A}$ shows a meta-analysis of time to first HHF from the five cardiovascular outcome studies with SGLT2 inhibitors showing this universal benefit for the class with no heterogeneity. In keeping with this, a recent meta-analysis of the association of SGLT2 inhibitors with cardiovascular and kidney outcomes in patients with type 2 diabetes concluded that the largest benefit across the class was for an associated reduction in risk for HHF and kidney outcomes, with benefits for HHF risk being the most consistent observation across the trials. ${ }^{15}$ Whilst the time to first MACE concludes a positive benefit for the class in metaanalysis (Figure 1B), there is nevertheless heterogeneity ${ }^{15}$ with VERTIS-CV7,8 and DECLARE-TIMI 58,13 being outliers. Figure 2 shows a meta-analysis of the two trials of patients with HFrEF (DAPA-HF6,14 and EMPEROR-Reduced ${ }^{9-11}$ ) which studied SGLT2 inhibitors in patients with and without diabetes. Using the primary outcome of first HHF or cardiovascular death, the same benefit is seen for dapagliflozin and empagliflozin in patients with and without diabetes. ${ }^{11,16}$ This suggests the benefit of SGLT2 inhibitors on heart failure does not depend upon the presence of diabetes or reduction in plasma glucose concentration, because SGLT2 inhibitors do not 
Figure 1. (A) Time to first hospitalisation for heart failure (HHF) and (B) time to first major adverse cardiovascular events (MACE) results from the five cardiovascular outcome trials with SGLT2 inhibitors shown side by side with result of meta-analysis also shown. The agents studied were empagliflozin (EMPA-REG OUTCOME), canagliflozin (CANVAS and CREDENCE), dapagliflozin (DECLARE-TIMI 58) and ertugliflozin (VERTIS-CV). Adapted from reference 34

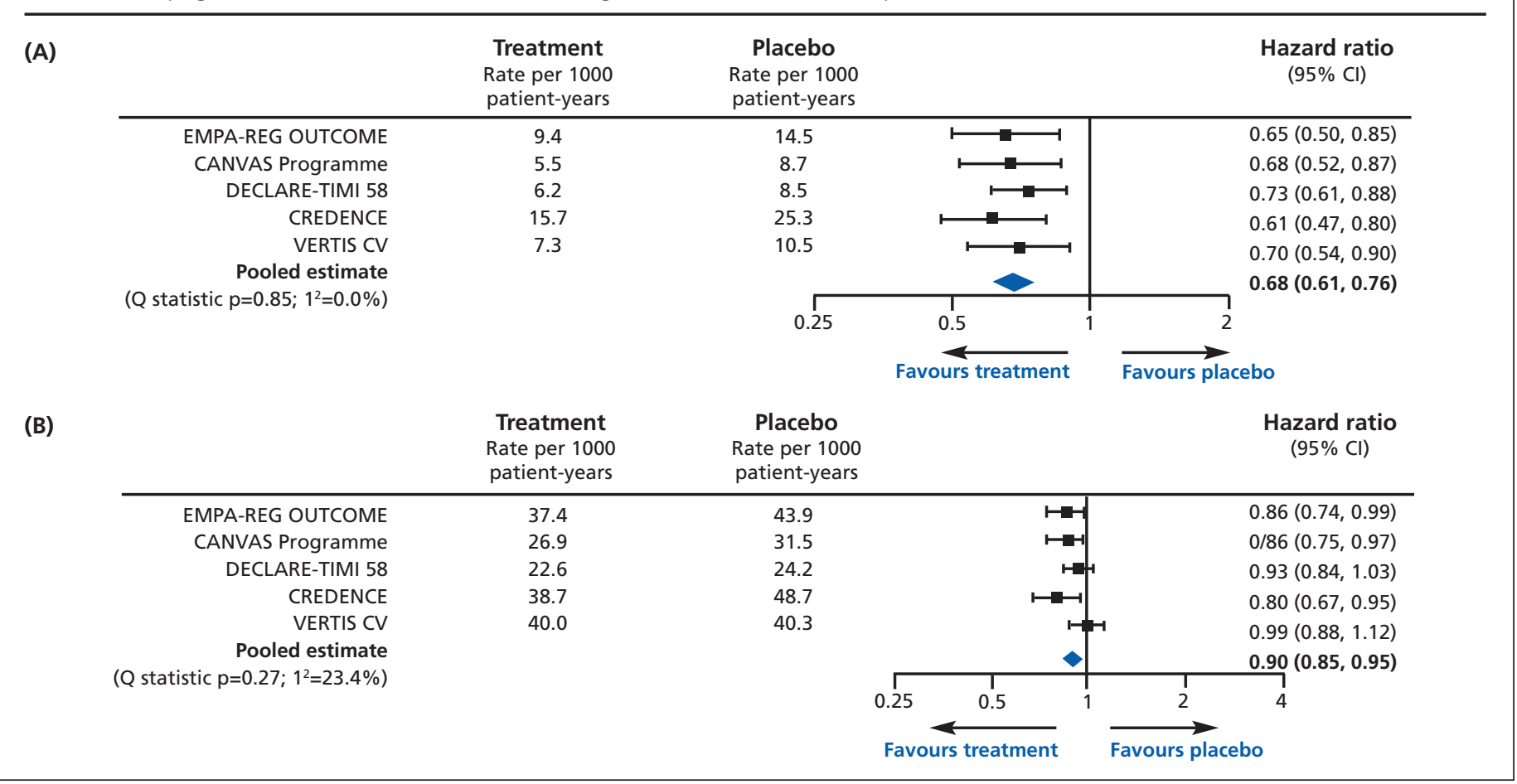

Figure 2. Time to first hospitalisation for heart failure or cardiovascular death results from the two trials which studied SGLT2 inhibitors in patients both with and without diabetes shown side by side with result of meta-analysis also shown. The agents studied were empagliflozin (EMPORER-Reduced) and dapagliflozin (DAPA-HF). Adapted from reference 11

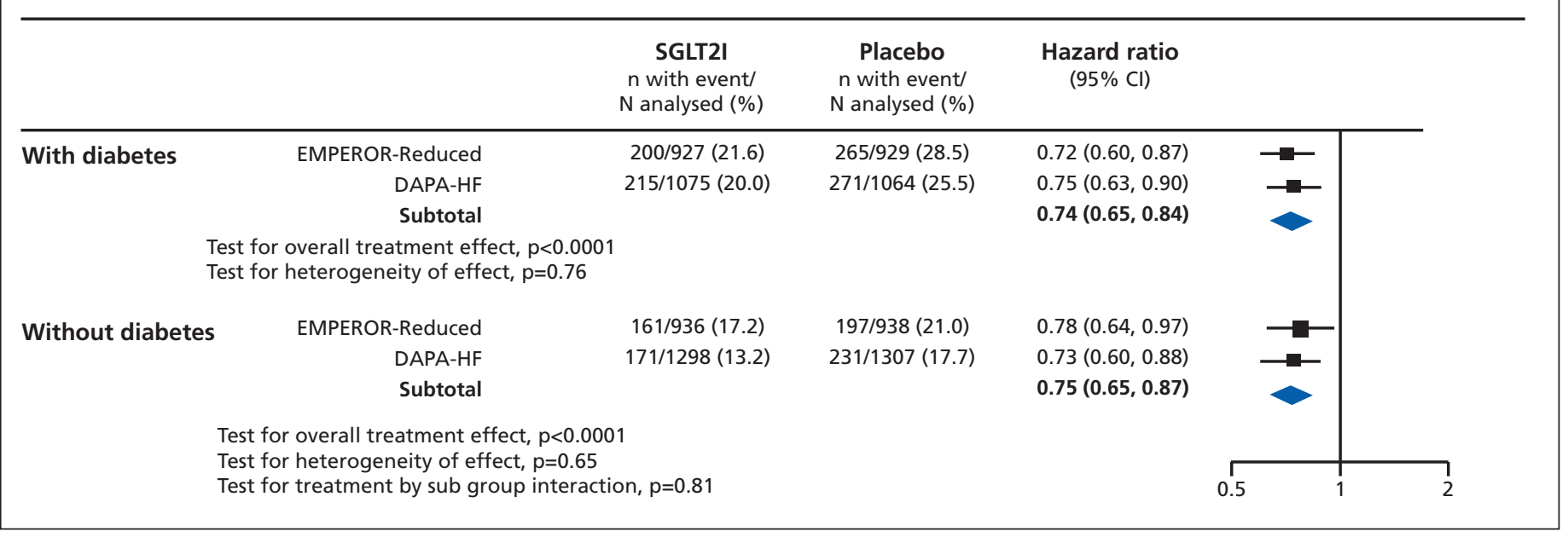

significantly lower fasting glucose concentration in non-diabetic individuals. ${ }^{17,18}$ Although at first sight it may seem noteworthy that the effect of empagliflozin on cardiovascular death in EMPORERReduced ( $\mathrm{HR}=0.92,95 \% \mathrm{Cl} 0.75$ to 1.12$)^{9-11}$ was non-significant compared with the significant effect of dapagliflozin in DAPA-HF $(\mathrm{HR}=0.82,95 \% \mathrm{Cl} 0.69$ to 0.98$),{ }^{11,14}$ the reverse was true when the effects on cardiovascular death were assessed in the cardiovascular outcome trials in comparable patients with type 2 diabetes ie. empagliflozin in EMPA-REG OUTCOME (HR=0.59, 95\% Cl 0.44 to 0.79$)^{1,19}$ and dapagliflozin in DECLARE-TIMI 58 (HR=0.92, 95\% $\mathrm{Cl} 0.69$ to 1.23). ${ }^{5,14}$ In contrast, the effects of these drugs to prevent HHF (and serious renal events - see below) seems to be a very consistent finding with this class of drugs. ${ }^{11}$ Several possible mechanisms by which SGLT2 inhibitors lead to this cardiac benefit in patients with HFrEF have been proposed, ${ }^{11,20,21}$ and these are summarised in Figure 3.

Whilst detailed consideration of the impact of SGLT2 inhibitors on renal disease is beyond the scope of this editorial, it is notewor- 
Figure 3. Possible mechanisms by which SGLT2 inhibitors exert their cardio-renal benefits. (A) Heart (B) Kidney. Adapted from references 20 and 21
(A)

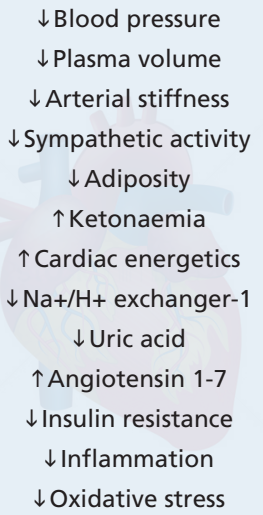

(B)

$\downarrow$ Blood pressure

$\uparrow$ Tubulo-glomerular feedback

$\downarrow$ Intra-glomerular pressure $\downarrow$ Hyperfiltration $\downarrow$ Inflammation

$\downarrow$ Oxidative stress $\downarrow$ Uric acid $\downarrow$ Glucotoxicity $\downarrow$ Adiposity $\triangle$ Renal energetics $\Delta$ Renal oxygenation thy that during 2020 it has become clearer than ever that SGLT2 inhibitors benefit renal outcomes. In Emperor-Reduced the annual rate of decline in the estimated glomerular filtration rate (eGFR) was slower in the empagliflozin group than in the placebo group $(-0.55$ vs $-2.28 \mathrm{~mL} / \mathrm{min} / 1.73 \mathrm{~m}^{2}$ body surface area per year, $\mathrm{p}<0.001$ ) and empagliflozin-treated patients had a lower risk of serious renal outcomes. $^{9-11}$ Meta-analysis with DAPA-HF showed that SGLT2 inhibition with empagliflozin and dapagliflozin reduced serious adverse renal outcomes. In a meta-analysis of trials in type 2 diabetes, SGLT2 inhibitors were associated with a reduced risk of kidney outcomes (HR=0.62, 95\% Cl 0.56 to 0.70). ${ }^{11}$ Trials of SGLT2 inhibitors in patients with established renal disease have also shown benefit for the class. In CREDENCE, canagliflozin reduced the relative risk of the renal-specific composite of end-stage kidney disease, a doubling of the creatinine level, or death from renal causes by $34 \%(H R=0.66$, $95 \% \mathrm{Cl} 0.53$ to 0.81 ), and the relative risk of end-stage kidney disease was lower by $32 \%$ (HR=0.68, $95 \% \mathrm{Cl} 0.54$ to 0.86 ). ${ }^{22}$ In DAPA$C K D$, dapagliflozin reduced the composite of sustained decline in the eGFR of at least $50 \%$, end-stage kidney disease, or death from renal causes by $46 \%$ ( $\mathrm{HR}=0.56,95 \% \mathrm{Cl} 0.45$ to 0.68$)$ - the renal benefit was seen regardless of the presence or absence of diabetes. ${ }^{23}$ Possible mechanisms by which SGLT2 inhibitors lead to renal benefit have been proposed ${ }^{11,20,21}$ and are summarised in Figure 3 . In summary, during the five years since the first presentation of EMPA-REG OUTCOME at the EASD in 2015, ${ }^{1}$ we now have six cardiovascular outcome trials, four in patients with type 2 diabetes (EMPA-REG, ${ }^{1,19}$ CANVAS, $^{3}$ DECLARE ${ }^{5,13}$ and VERTIS-CV 7,8 ) and two in patients both with and without diabetes (DAPA-HF 6 ,14 and EMPEROR-Reduced ${ }^{9-11}$ ) of four SGLT2 inhibitors with time to first HHF plus $\mathrm{CV}$ death being the primary outcome in two trials $6,9-11$ and two renal outcome trials (CREDENCE ${ }^{22}$ and DAPA-CKD' ${ }^{23}$ ) that have demonstrated cardiovascular safety and cardiovascular efficacy for selected outcomes. Meta-analysis results demonstrate moderate heterogeneity across the class/trials for MACE and cardiovascular death and remarkable consistency amongst all eight trials regarding heart failure. Two trials have demonstrated the safety and efficacy of dapagliflozin and empagliflozin for treatment of HFrEF.,9,-11

\section{GLP-1 receptor agonists}

It was noteworthy that the MACE cardiovascular benefit of the longacting GLP-1RA, dulaglutide, in REWIND was primarily driven by a benefit on stroke reduction (by $24 \% ; H R=0.76,95 \% \mathrm{CI} 0.62$ to 0.94). ${ }^{24}$ In all the other trials of long-acting GLP1-RAs, LEADER (liraglutide), ${ }^{2}$ SUSTAIN-6 (semaglutide), ${ }^{5}$ EXSCEL (exenatide QW), ${ }^{4} \mathrm{Har}-$ mony Outcomes (albiglutide) ${ }^{5}$ and PIONEER 6 (oral semaglutide) ${ }^{6}$ the number of strokes was less in the treatment group than in the placebo group, although in the individual studies this did not achieve statistical significance. Meta-analysis of the seven cardiovascular outcome trials with GLP-1RAs showed a $16 \%$ reduction in stroke for the class as a whole ( $\mathrm{HR}=0.84,95 \% \mathrm{Cl} 0.76$ to 0.93 ; Figure 4). ${ }^{25}$ Against this background, in the post hoc analysis from the LEADER, SUSTAIN 6 and PIONEER 6 trials, data were pooled to examine the effect of liraglutide and semaglutide on stroke and its subtypes. ${ }^{12}$ Across the three trials, $216 / 7,907$ (2.7\%) in the GLP1RA group and 262/7,913 (3.3\%) in the placebo group had a history of stroke. There was an $18 \%$ reduction in the time to first occurrence of stroke in the GLP-1RA group compared with the placebo group (HR=0.82, 95\% Cl 0.68 to 0.98 ; Figure $5 A$ ). Treatment effects were consistent across all stroke subtypes. However,

Figure 4. Fatal or non-fatal stroke results from the seven cardiovascular outcome trials with GLP-1RAs shown side by side with result of meta-analysis also shown. The names of the studies and the agents studied are shown. For more information on these studies see references 2,4-6,24,25,35. Adapted from references 25 and 35.

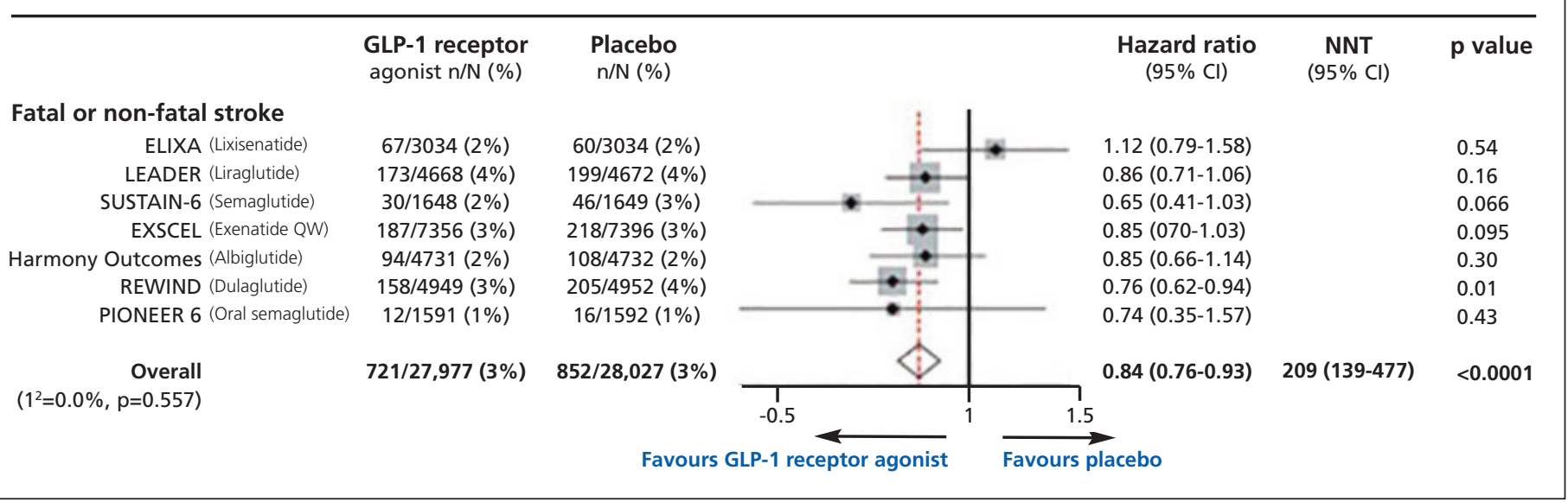


Figure 5. Cumulative incidence of stroke over time in patients treated with GLP-1 receptor analogues versus placebo group. (A) Liraglutide and semaglutide in the LEADER, SUSTAIN 6 and PIONEER 6 trials. (B) Semaglutide in the SUSTAIN 6 and PIONEER 6 trials. Adapted from reference 12.

(A)

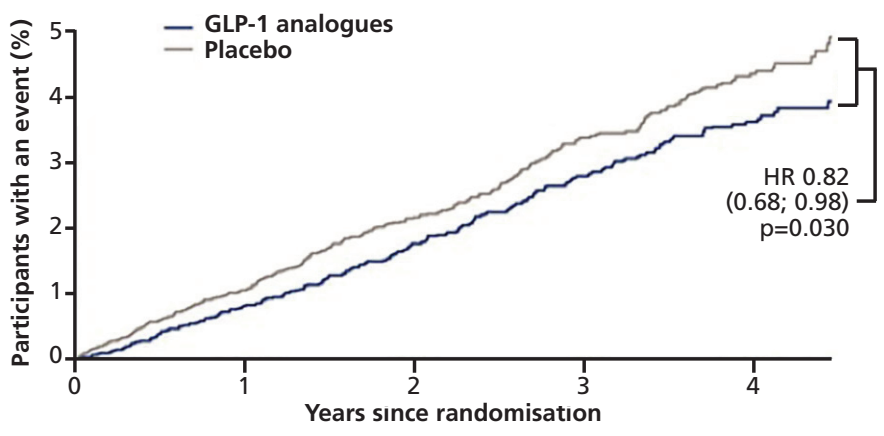

(B)

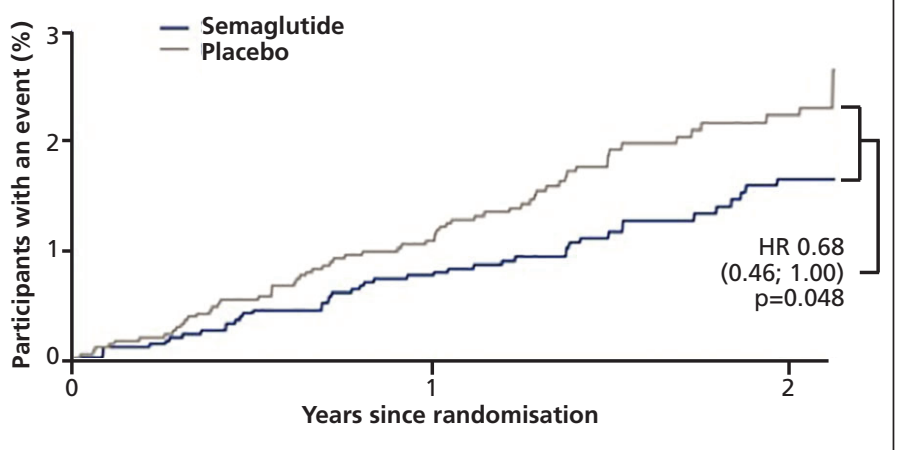

the greatest benefit was seen regarding small vessel occlusion strokes $(\mathrm{HR}=0.78,95 \% \mathrm{Cl} 0.59$ to $1.02, \mathrm{p}=0.07)$. The impact of semaglutide seemed to be greater than liraglutide, with a significant reduction in outcomes despite smaller and shorter trials (Figure 5B). It is noteworthy that separation of the Kaplan-Meier curves occurred very early (Figures $5 \mathrm{~A}$ and $5 \mathrm{~B}$ ) and hence it seems likely that at least some of the benefit is via mechanisms other than reducing atherosclerosis. ${ }^{12}$

The case for combination therapy now stronger than ever In previous editorials we proposed that SGLT2 inhibitors, long-acting GLP-1RAs, pioglitazone and metformin in combination could complement each other to prevent cardiovascular events and save lives in patients with type 2 diabetes at high cardiovascular risk. ${ }^{1-6}$ With regard to stroke, we came to this conclusion because of the accumulated evidence from multiple studies suggesting that pioglitazone is very effective in reducing stroke risk by slowing down - or even reversing - the atherosclerotic process ${ }^{1-3,26-28}$ whereas, as discussed above, the cardiovascular benefit of SGLT2 inhibitors is primarily due to a reduction in cardiac risk with little benefit on stroke risk and seems not to be mediated via slowing/reduction of atherosclerosis. ${ }^{11,20,21}$ On the other hand, although both GLP-1RAs and pioglitazone significantly reduce the risk of stroke, the cellular/molecular mechanisms of GLP-1RAs and pioglitazone are distinct (GLP-1 receptor activation versus PPAR gamma activation). ${ }^{5}$ Further, as previously noted, SGLT2 inhibitors mitigate the fluid retention associated with pioglitazone use, ${ }^{29}$ suggesting that pioglitazone and SGLT2 inhibitors would complement each other not only in reducing cardiovascular risk but also in reducing side effects related to fluid retention. We pointed to the evidence that the early use of triple combination therapy with metformin, pioglitazone and a GLP-1RA achieved lower $\mathrm{HbA}_{1 c}$, weight loss and much less hypoglycaemia compared with the traditional approach of sequential escalation through metformin, sulfonylurea and insulin, which was associated with significant weight gain. ${ }^{30}$

From the new evidence gained in 2020 we can now expand these previous editorials:

- It is clear, as discussed above, that SGLT2 inhibitors provide their benefit through improvement in, and protection from, heart failure and complement pioglitazone by reducing the side effects of the thiazolidinedione which reduces atherosclerosis. ${ }^{1-3,26-28}$ Because the SGLT2 inhibitors and pioglitazone work via entirely different mechanisms, it is likely that the two medications combined would have an additive cardiovascular protective effect. A cardiovascular outcome study in patients with an established history of cardiovascular disease comparing the combination of an SGLT2 inhibitor with pioglitazone versus SGLT2 inhibitor alone would be of great interest. In this context it is reassuring that, in the PROactive study, although the incidence of serious heart failure was increased with pioglitazone versus placebo in the total PROactive population of patients with type 2 diabetes and macrovascular disease, subsequent mortality or morbidity was not increased in patients with serious heart failure. ${ }^{31}$ Furthermore, as we have discussed above, it seems likely that SGLT2 inhibitors would mitigate further any increase in heart failure associated with pioglitazone. ${ }^{29}$

- Multiple mechanisms have been suggested regarding the cardiovascular benefit of GLP-1RAs. ${ }^{5}$ Because of the early separation of the curves by GLP-1RAs, it is likely that mechanisms additional to atherosclerosis are activated by GLP-1RAs and benefit stroke (Figures 5A and 5B). ${ }^{12}$ Further, because of the distinct cellular/molecular mechanism of GLP-1RA and pioglitazone action, it is possible that a combination of both agents will exert an additive benefit to slow atherosclerosis and reduce stroke risk. ${ }^{1-3,26-28}$ It is noteworthy that the benefit of stroke in both the IRIS study ${ }^{32}$ and the PROactive study ${ }^{33}$ was relatively rapid and the difference between the two curves continued to widen thereafter. Thus, it is likely that GLP-1RAs and pioglitazone exert an additive cardiovascular benefit, particularly for stroke reduction. A cardiovascular outcome trial looking particularly at stroke, comparing the combination of dulaglutide or semaglutide with pioglitazone versus either medication alone, especially with regard to stroke, would be of great interest.

Conflict of interest REJR: speaker fees and/or consultancy fees and/or educational sponsorships from AstraZeneca, BioQuest, GI Dynamics, Janssen, Novo Nordisk, Sanofi-Aventis and Takeda. RAD: Advisory board: AstraZeneca, Novo Nordisk, Janssen, Boehringer-Ingelheim, Intarcia. Research support: Boehringer-Ingelheim, AstraZeneca, Janssen, Merck - Research Grant (Investigator). Speaker's bureau: Novo Nordisk, AstraZeneca - Honorarium (Speaker). MAA has nothing to disclose

Funding None. 


\section{References}

1. Ryder REJ, DeFronzo RA. Diabetes medications with cardiovascular protection in the wake of EMPA-REG OUTCOMETM: the optimal combination may be metformin, pioglitazone and empagliflozin. $\mathrm{Br}$ J Diabetes Vasc Dis 2015; 15:151-4. http://dx.doi.org/10.15277/bjdvd.2015.045

2. Ryder REJ, DeFronzo RA. Diabetes medications with cardiovascular protection - what now after LEADER $^{\circledR}$ ? Could metformin, pioglitazone, empagliflozin and liraglutide complement each other to save lives? $\mathrm{Br} J$ Diabetes 2016;16:103-6. http://dx.doi.org/10.15277/bjd.2016.096

3. Ryder REJ, DeFronzo RA. What now on the CANVAS of diabetes medications with cardiovascular protection? Could metformin, pioglitazone, SGLT2 inhibitors and liraglutide complement each other to save lives? Br J Diabetes 2017:17:89-92. http://dx.doi.org/10.15277/bjd.2017.036

4. Ryder REJ, DeFronzo RA. Diabetes medications with cardiovascular protection in the wake of EXSCEL - is there a class effect for long-acting GLP-1 receptor agonists? Br J Diabetes 2017;17:131-3. https://doi.org/10.15277/ bjd.2017.147

5. Ryder REJ, DeFronzo RA. Diabetes medications with cardiovascular protection after HARMONY Outcomes and DECLARE-TIMI 58: could metformin, pioglitazone, SGLT2 inhibitors and long-acting GLP-1 receptor agonists complement each other to save lives by different mechanisms? Br J Diabetes 2019;19:1-5. https://doi.org/10.15277/bjd.2019.207

6. Ryder REJ, DeFronzo RA. Diabetes medications with cardiovascular protection as we enter a new decade: can SGLT2 inhibitors, long-acting GLP-1 receptor agonists, pioglitazone and metformin complement each other to save lives? Br J Diabetes 2020;20:5-8. https://doi.org/10.15277/bjd.2020.250

7. Results of the eValuation of ERTugliflozin Efflcacy and Safety CardioVascular Outcomes Trial (VERTIS-CV). Presented on 16 June 2020 during the 80th Scientific Sessions of the American Diabetes Association virtual meeting. Slides available at https://www.acc.org/education-and-meetings/image-andslide-gallery/media-detail?id=307A7E103BC04A588A3370709253FC35 (accessed 14 October 2020)

8. Cannon CP, Pratley R, Dagogo-Jack S, et al. Cardiovascular outcomes with ertugliflozin in type 2 diabetes. N Engl J Med 2020;383:1425-35. https://doi.org/10.1056/NEJMoa2004967

9. EMPEROR-Reduced meets primary endpoint in heart failure with reduced ejection fraction. Report of a Hot Line Session at European Society of Cardiology Congress 2020 - The Digital Experience. https://www.escardio.org/ The-ESC/Press-Office/Press-releases/EMPEROR (accessed 14 October 2020).

10. Packer M, Anker SD, Butler J, et al. Cardiovascular and renal outcomes with empagliflozin in heart failure. N Engl J Med 2020;383:1413-24. https://doi.org/10.1056/NEJMoa2022190

11. EASD VIRTUAL MEETING 2020. Webcast: Empagliflozin for the treatment of chronic heart failure and a reduced ejection fraction in patients with and without diabetes: new results of the EMPEROR-Reduced trial. https://www.easd.org/virtualmeeting2020/\#! contentsessions/3384 (accessed 14 October 2020)

12. Strain WD, Holst AG, Rasmussen $S$, et al. Effects of liraglutide and semaglutide on stroke subtypes in patients with type 2 diabetes: a post hoc analysis of the LEADER, SUSTAIN 6 and PIONEER 6 trials. EASD abstract 258 in OP47 at https://link.springer.com/article/10.1007/s00125-020-05221-5\#Sec47 (accessed 15 October 2020). Webcast at https://www.easd.org/virtualmeeting2020/\#! resources/effects-of-liraglutide-and-semaglutide-on-stroke-subty pes-in-patients-with-type-2-diabetes-a-post-hoc-analysis-of-the-leader-sustain-6-and-pioneer-6-trials (accessed 15 October 2020).

13. Wiviott SD, Raz I, Bonaca MP, et al. Dapagliflozin and cardiovascular outcomes in type 2 diabetes. N Engl J Med 2019;380:347-57. https://doi.org/ 10.1056/NEJMoa1812389

14. McMurray JJV, Solomon SD, Inzucchi SE, et al. Dapagliflozin in patients with heart failure and reduced ejection fraction. N Engl J Med 2019;381:19952008. https://doi.org/10.1056/NEJMoa1911303

15. McGuire DK, Shih WJ, Cosentino F, et al. Association of SGLT2 Inhibitors with cardiovascular and kidney outcomes in patients with type 2 diabetes: a metaanalysis. JAMA Cardiol 2020 Oct 7;e204511. https://doi.org/10.1001/jamacardio.2020.4511 [Online ahead of print].

16. Zannad F, Ferreira JP, Pocock SJ, et al. SGLT2 inhibitors in patients with heart failure with reduced ejection fraction: a meta-analysis of the EMPERORReduced and DAPA-HF trials. Lancet 2020;396(10254):819-29. https://doi.org/10.1016/S0140-6736(20)31824-9

17. Al Jobori H, Daniele G, Adams J, et al. Determinants of the increase in ketone concentration during SGLT2 inhibition in NGT, IFG and T2DM patients. Diabetes Obes Metab 2017;19:809-13. https://doi.org/10.1111/dom.12881
18. Komoroski B, Vachharajani N, Boulton D, et al. Dapagliflozin, a novel SGLT2 inhibitor, induces dose-dependent glucosuria in healthy subjects. Clin Pharmacol Ther 2009:85:520-6. https://doi.org/10.1038/clpt.2008.251

19. Zinman B, Wanner C, Lachin JM, et al. Empagliflozin, cardiovascular outcomes and mortality in type 2 diabetes. N Engl J Med 2015;373:2117-28. http://dx.doi.org/10.1056/NEJMoa1504720

20. DeFronzo R, Norton L, Abdul-Ghani M. Renal, metabolic and cardiovascular considerations of SGLT2 inhibition. Nat Rev Nephrol 2017;13:11-26. https://doi.org/10.1038/nrneph.2016.170

21. Bailey CJ. EASD VIRTUAL MEETING 2020. Webcast commentary on empagliflozin for the treatment of chronic heart failure and a reduced ejection fraction in patients with and without diabetes: new results of the EMPERORReduced trial. https://www.easd.org/virtualmeeting2020/\#!resources/commentary-e5e86c86-9a96-4f71-aee8-665b616ebbb6 (accessed 15 October 2020).

22. Perkovic V, Jardine MJ, Neal B, et al. Canagliflozin and renal outcomes in type 2 diabetes and nephropathy. N Engl J Med 2019;380:2295-306. https://doi.org/10.1056/NEJMoa1811744

23. Heerspink HJL, Stefánsson BV, Correa-Rotter R, et al. Dapagliflozin in patients with chronic kidney disease. N Engl J Med 2020;383:1436-46. https://doi.org/10.1056/NEJMoa2024816

24. Gerstein HC, Colhoun HM, Dagenais GR, et al. Dulaglutide and cardiovascular outcomes in type 2 diabetes (REWIND): a double-blind, randomised placebo-controlled trial. Lancet 2019;394:121-30. https://doi.org/10.1016/ S0140-6736(19)31149-3

25. Kristensen SL, Rørth R, Jhund PS, et al. Cardiovascular, mortality, and kidney outcomes with GLP-1 receptor agonists in patients with type 2 diabetes: a systematic review and meta-analysis of cardiovascular outcome trials. Lancet Diabetes Endocrinol 2019;7:776-85. https://doi.org/10.1016/S22138587(19)30249-9

26. Ryder REJ. Pioglitazone has a dubious bladder cancer risk but an undoubted cardiovascular benefit. Diabet Med 2015;32:305-13. http://dx.doi.org/ 10.1111/dme.12627

27. Mazzone T, Meyer PM, Feinstein SB, et al. Effect of pioglitazone compared with glimepiride on carotid intima-media thickness in type 2 diabetes: a randomized trial. JAMA 2006;296:2572-81. http://dx.doi.org/10.1001/ jama.296.21.joc60158

28. Nissen SE, Nicholls SJ, Wolski $\mathrm{K}$, et al. Comparison of pioglitazone vs glimepiride on progression of coronary atherosclerosis in patients with type 2 diabetes: the PERISCOPE randomized controlled trial. JAMA 2008;299: 1561-73. http://dx.doi.org/10.1001/jama.299.13.1561

29. Gautam A, Agrawal PK, Prakash P, Hazra DK. Pioglitazone associated pedal edema resolved by adding sodium glucose co-transporter 2 inhibitor. ADA 76th Scientific Sessions 2016. Late Breaking Poster Session, Poster 140-LB. Abstract 140-LB. Available at https://diabetes.diabetesjournals.org/content/ diabetes/suppl/2016/06/20/65.Supplement_1.DC 1/2016_ADA_LB_Abstracts HiRes FINAL 5 11 16.pdf (accessed 15 October 2020).

30. Abdul-Ghani MA, Puckett $\bar{C}$, Triplitt C, et al. Initial combination therapy with metformin, pioglitazone and exenatide is more effective than sequential add-on therapy in subjects with new-onset diabetes. Results from the Efficacy and Durability of Initial Combination Therapy for Type 2 Diabetes (EDICT): a randomized trial. Diabetes Obes Metab 2015;17:268-75. https://doi.org/10.1111/dom.12417

31. Erdmann E, Charbonnel B, Wilcox RG, et al. Pioglitazone use and heart failure in patients with type 2 diabetes and preexisting cardiovascular disease: data from the PROactive study (PROactive 08). Diabetes Care 2007; 30:2773-8. https://doi.org/10.2337/dc07-0717

32. Kernan WN, Viscoli CM, Furie KL, et al; IRIS Trial Investigators. Pioglitazone after ischemic stroke or transient ischemic attack. N Engl J Med 2016;374: 1321-31. https://doi.org/10.1056/NEJMoa1506930

33. Wilcox R, Bousser MG, Betteridge DJ, et al. Effects of pioglitazone in patients with type 2 diabetes with or without previous stroke: results from PROactive (PROspectivepioglitAzone Clinical Trial In macroVascular Events 04). Stroke 2007;38:865-73. https://doi.org/10.1161/01.STR.0000257974.06317.49

34. EASD VIRTUAL MEETING 2020. Webcast: VERTIS-CV outcome. https://www.easd.org/virtualmeeting/home.html\#! contentsessions/3380 (accessed 19 Novemberbob 2020).

35. Oral Semaglutide-The PIONEER Program Trials. Presented on 11 June 2019 during the 79th Scientific Sessions of the American Diabetes Association in San Francisco, USA. Slides available at https://tracs.unc.edu/PIONEER (accessed 12 February 2020). 